\title{
A new approach to ticagrelor-based de-escalation of antiplatelet therapy after acute coronary syndrome. A rationale for a randomized, double-blind, placebo-controlled, investigator-initiated, multicenter clinical study
}

Jacek Kubica ${ }^{1}$, Piotr Adamski ${ }^{1}$, Piotr Niezgoda ${ }^{1}$, Aldona Kubica ${ }^{2}$, Przemysław Podhajski ${ }^{1}$, Malwina Barańska ${ }^{1}$, Julia M. Umińska ${ }^{3}$, Łukasz Pietrzykowski ${ }^{2}$, Małgorzata Ostrowska ${ }^{1}$, Jolanta M. Siller-Matula, ${ }^{4}$, Jolita Badariené ${ }^{6,7}$, Stanisław Bartuśs ${ }^{8}$, Andrzej Budaj, Sławomir Dobrzycki ${ }^{10}$, Łukasz Fidor ${ }^{11}$, Mariusz Gąsior ${ }^{12}$, Jacek Gessek ${ }^{13}$, Marek Gierlotka ${ }^{14}$, Robert Gil ${ }^{15}$, Jarosław Gorący ${ }^{16}$, Paweł Grzelakowski ${ }^{17}$, Tomasz Hajdukiewicz ${ }^{18}$, Miłosz Jaguszewski ${ }^{19}$, Marianna Janion ${ }^{20}$, Jarosław Kasprzak ${ }^{21}$, Adam Kern ${ }^{22,23}$, Artur Klecha ${ }^{24}$, Andrzej Kleinrok ${ }^{25,26}$, Wacław Kochman ${ }^{27}$, Bartosz Krakowiak $^{28}$, Jacek Legutko ${ }^{29}$, Maciej Lesiak ${ }^{30}$, Marcin Nosal $^{31}$, Grzegorz Piotrowski ${ }^{32}$, Andrzej Przybylski ${ }^{33}$, Tomasz Roleder ${ }^{34}$, Grzegorz Skonieczny ${ }^{35}$, Grzegorz Sobieszek ${ }^{36}$, Agnieszka Tycińska ${ }^{37}$, Dariusz Wojciechowski ${ }^{38}$,

Wojciech Wojakowski ${ }^{39}$, Jarosław Wójcik ${ }^{40}$, Marzenna Zielińska ${ }^{41}$, Aleksander Żurakowski ${ }^{42}$, Giuseppe Specchia ${ }^{43}$, Diana A. Gorog ${ }^{44,45}$, Eliano P. Navarese ${ }^{1}$

${ }^{1}$ Department of Cardiology and Internal Medicine, Collegium Medicum, Nicolaus Copernicus University, Bydgoszcz, Poland; ${ }^{2}$ Department of Health Promotion, Collegium Medicum, Nicolaus Copernicus University, Bydgoszcz, Poland; ${ }^{3}$ Department of Geriatrics, Collegium Medicum, Nicolaus Copernicus University,

Bydgoszcz, Poland; ${ }^{4}$ Department of Experimental and Clinical Pharmacology, Medical University of Warsaw, Poland; ${ }^{5}$ Department of Cardiology, Medical University of Vienna, Austria; ${ }^{6}$ Clinic of Cardiac and Vascular Diseases, Institute of Clinical Medicine, Faculty of Medicine, Vilnius University, Vilnius, Lithuania; ${ }^{7}$ Center of Cardiology and Angiology, Vilnius University Hospital Santaros Klinikos, Vilnius, Lithuania; ${ }^{8}$ Department of Cardiology and Cardiovascular Interventions, University Hospital, Krakow, Poland; ${ }^{9}$ Department of Cardiology, Center of Postgraduate Medical Education, Grochowski Hospital,

Warsaw, Poland; ${ }^{10}$ Department of Invasive Cardiology, University Hospital, Bialystok, Poland;

${ }^{11}$ Department of Cardiology, Tuchola Hospital, Tuchola, Poland; ${ }^{12} 3^{\text {rd }}$ Department of Cardiology, Silesian Center for Heart Diseases, Faculty of Medicine in Zabrze, Medical University of Silesia, Zabrze, Poland;

${ }^{13}$ Department of Cardiology and Intensive Cardiological Care, Specialized Municipal Hospital, Torun,

Poland; ${ }^{14}$ Department of Cardiology, University Hospital, Institute of Medical Sciences, University of Opole, Poland; ${ }^{15}$ Department of Invasive Cardiology, Central Clinical Hospital MSWiA, Warsaw, Poland; ${ }^{16}$ Department of Cardiology, University Hospital No. 2, Pomeranian Medical University, Szczecin, Poland;

${ }^{17}$ Department of Cardiology and Cardiac Surgery, $10^{\text {th }}$ Military Research Hospital and Polyclinic,

Bydgoszcz, Poland; ${ }^{18}$ Department of Cardiology, Provincial Hospital, Elblag, Poland;

Address for correspondence: Dr. Piotr Niezgoda, Department of Cardiology and Internal Medicine, Collegium Medicum, Nicolaus Copernicus University, ul. M. Skłodowskiej-Curie 9, 85-094 Bydgoszcz, Poland, tel: +48 525854023 , fax: +4852585 4024, e-mail: piotr.niezgoda1986@gmail.com

Received: 24.03.2021 Accepted: 7.04.2021

This article is available in open access under Creative Common Attribution-Non-Commercial-No Derivatives 4.0 International (CC BY-NC-ND 4.0) license, allowing to download articles and share them with others as long as they credit the authors and the publisher, but without permission to change them in any way or use them commercially. 
${ }^{19} 1^{\text {st }}$ Department of Cardiology, Medical University of Gdansk, Poland; ${ }^{20}$ Institute of Medical Sciences, Collegium Medicum, Jan Kochanowski University, Kielce, Poland; ${ }^{21} 1^{\text {st }}$ Department and Chair of Cardiology, Medical University of Lodz, Bieganski Hospital, Lodz, Poland; ${ }^{22}$ Department of Cardiology and Internal Medicine, Medical Faculty, University of Warmia and Mazury in Olsztyn, Poland; ${ }^{23}$ Department of Cardiology, Voivodal Specialist Hospital in Olsztyn, Poland; ${ }^{24}$ Department of Cardiology, Podhalanski Specialized Hospital, Nowy Targ, Poland; ${ }^{25}$ University of Information Technology and Management in Rzeszow, Poland; ${ }^{26}$ Department of Cardiology, The Pope John Paul II Hospital in Zamosc, Poland;

${ }^{27}$ The National Institute of Cardiology, Department of Cardiology, Bielanski Hospital, Warsaw, Poland; ${ }^{28}$ Department of Cardiology, Center for Heart Diseases, Military Hospital, Wroclaw, Poland; ${ }^{29}$ Jagiellonian University Medical College, Institute of Cardiology, Department of Interventional Cardiology, The John Paul II Hospital in Krakow, Poland; ${ }^{30}$ Chair and $1^{\text {st }}$ Department of Cardiology, Poznan University of Medical Sciences, Poznan, Poland; ${ }^{31}$ Center for Invasive Cardiology, Electrotherapy and Angiology, Krosno, Poland; ${ }^{32}$ Department of Cardio-oncology, Medical University of Lodz, Poland; ${ }^{33}$ Medical College University of Rzeszow, Poland; ${ }^{34}$ Department of Cardiology, Provincial Hospital in Wroclaw, Poland; ${ }^{35}$ Department of Cardiology and Intensive Cardiological Care, Rydygier Provincial Hospital, Torun, Poland; ${ }^{36}$ Department of Cardiology, $1^{\text {st }}$ Military Hospital, Lublin, Poland; ${ }^{37}$ Department of Cardiology, Medical University of Bialystok, Poland; ${ }^{38}$ Department of Cardiology, Wolski Hospital, Warsaw, Poland; ${ }^{39}$ Division of Cardiology and Structural Heart Diseases, Medical University of Silesia, Katowice, Poland; ${ }^{40}$ Center for Invasive Cardiology IKARDIA, Naleczow, Poland; ${ }^{41}$ Department of Interventional Cardiology, Medical University of Lodz, Poland; ${ }^{42}$ Malopolska Cardiovascular Center of Polish-American Heart Clinics, Chrzanow, Poland; ${ }^{43}$ Pavia, Italy; ${ }^{44}$ Postgraduate Medical School, University of Hertfordshire, Stevenage, United Kingdom;

${ }^{45}$ Faculty of Medicine, National Heart and Lung Institute, Imperial College, London, United Kingdom

\title{
This paper was guest edited by Prof. Stefan Grajek
}

\begin{abstract}
The risk of ischemic events gradually decreases after acute coronary syndrome (ACS), reaching a stable level after 1 month, while the risk of bleeding remains steady during the whole period of dual antiplatelet treatment (DAPT). Several de-escalation strategies of antiplatelet treatment aiming to enhance safety of $D A P T$ without depriving it of its efficacy have been evaluated so far.

We hypothesized that reduction of the ticagrelor maintenance dose 1 month after ACS and its continuation until 12 months after ACS may improve adherence to antiplatelet treatment due to better tolerability compared with the standard dose of ticagrelor. Moreover, improved safety of treatment and preserved anti-ischemic benefit may also be expected with additional acetylsalicylic acid (ASA) withdrawal. To evaluate these hypotheses, we designed the Evaluating Safety and Efficacy of Two Ticagrelor-based De-escalation Antiplatelet Strategies in Acute Coronary Syndrome - a randomized clinical trial (ELECTRA-SIRIO 2), to assess the influence of ticagrelor dose reduction with or without continuation of ASA versus DAPT with standard dose ticagrelor in reducing clinically relevant bleeding and maintaining anti-ischemic efficacy in ACS patients.

The study was designed as a phase III, randomized, multicenter, double-blind, investigator-initiated clinical study with a 12-month follow-up (ClinicalTrials.gov Identifier: NCT04718025; EudraCT number: 2020-005130-15). (Cardiol J 2021; 28, 4: 607-614)
\end{abstract}

Key words: acute coronary syndrome, ticagrelor, antiplatelet therapy, de-escalation, ELECTRA-SIRIO 2

\section{Introduction}

Elevated rates of ischemic events clustering during the first month after acute coronary syndrome (ACS) is reflective of elevated platelet reactivity during this period. The risk of major adverse cardiovascular events (MACE), including cardiovascular death, myocardial infarction (MI), and stroke, gradually decreases, reaching a stable level after 1 month, while the risk of bleeding is 
mainly related to the type and dosage of antiplatelet drugs and remains steady during the whole period of dual antiplatelet treatment (DAPT). Therefore, in the earliest phase after ACS, the ischemic component should be especially targeted with potent antiplatelet strategies, whereas after the clinical stabilization occurs, de-escalation of the antiplatelet therapy may be justified [1-8]. Treatment with ticagrelor or prasugrel is recommended over clopidogrel due to better efficacy, albeit with more bleeding complications. These higher bleeding rates have provoked trials investigating de-escalation from ticagrelor or prasugrel to clopidogrel in the hope of reducing bleeding without increasing rates of thrombotic events $[4,5]$. Several strategies aiming to enhance the safety of antiplatelet treatment without depriving it of its efficacy have been evaluated so far.

Replacing a potent P2Y12 receptor inhibitor (prasugrel or ticagrelor) with a weaker one (clopidogrel) was the first de-escalation strategy tested. In the TOPIC study downgrading of DAPT (from prasugrel or ticagrelor to clopidogrel) 1 month after ACS was associated with a net clinical benefit mainly driven by a significant reduction in bleeding complications, while the risk of recurrent ischemic events remained unchanged [9]. The limitations of this study, including small study sample size (645 patients), non-homogenous treatment at baseline and in the control arm (prasugrel or ticagrelor), and moderate adherence to the treatment, warrant cautious interpretation of these results. On the other hand, the SCOPE registry $(n=1363)$ reported switching from clopidogrel to novel P2Y12 receptor inhibitors to be safe, while a downgrade was an independent predictor of net adverse cerebrovascular events in patients with ACS [10]. The safety and efficacy of early de-escalation of antiplatelet treatment from prasugrel to clopidogrel were tested in the TROPICAL-ACS study $(\mathrm{n}=2610)$ [11]. High platelet reactivity is associated with an increased risk of recurrent ischemic events, while the use of clopidogrel is burdened with high interindividual variability of the antiplatelet effect and the possibility of drug interactions [12]. Hence, platelet function testing was applied for guidance of de-escalation of antiplatelet treatment in this study. In the de-escalation group as much as $39 \%$ of patients required a switch-back to prasugrel due to insufficient platelet inhibition with clopidogrel defined as high platelet reactivity [11]. The primary endpoint, defined as net clinical benefit (cardiovascular death, MI, stroke, or bleeding grade 2 or higher according to Bleeding Academic Research
Consortium [BARC] criteria) 1 year after randomization, occurred in $7 \%$ of patients in the guided deescalation group and in $9 \%$ of patients in the control group $\left(\mathrm{p}_{\text {non-inferiority }}=0.0004\right.$; hazard ratio $[\mathrm{HR}] 0.81$; $95 \%$ confidence interval $[\mathrm{CI}] 0.62-1.06, \mathrm{p}_{\text {superiority }}$ $=0.12$ ). The prevalence of ischemic events (cardiovascular death, MI, and stroke) was $3 \%$ in both study arms, while the prevalence of BARC grade 2 or higher was $5 \%$ and $6 \%$, respectively. Despite of the lack of significant differences in the whole study population, subgroup analyses revealed a net clinical benefit from guided de-escalation in ST-segment elevation MI patients, subjects $\leq 70$ years of age, and those without diabetes. Summing up, this investigator-initiated, randomized, open-label, assessor-blinded, multicenter trial showed that platelet function testing-guided de-escalation from prasugrel to clopidogrel was non-inferior to standard treatment with prasugrel at 1 year after percutaneous coronary intervention (PCI) in terms of net clinical benefit in patients with ACS [11].

Kheiri et al. [13] conducted a meta-analysis (3 randomized clinical trials with 3391 patients) aimed at evaluating the clinical outcomes of antiplatelet de-escalation based on switching from prasugrel or ticagrelor to clopidogrel compared with continuation of DAPT with more potent P2Y12 receptor inhibitor in patients treated with PCI. The net clinical outcome (composite of bleeding or thrombotic events) was significantly reduced in the group switched to clopidogrel; however, no differences between the groups in a separate analysis for MACE, as well as for bleedings, were found [13].

Pharmacodynamic data show that reduction of ticagrelor bioavailability by one-third significantly decreases the antiplatelet effect of ticagrelor in patients with acute MI, but not in subjects without ACS [14-16]. More pronounced ticagrelor-induced platelet inhibition seen in a stable setting compared with MI reflects the excessive platelet activation occurring during the initial phase of ACS. Subsequently, the enhanced platelet reactivity and aggregation decrease over time when ACS patients become stable [17-22]. This observation was confirmed in a sub-study of the PEGASUS-TIMI 54 trial showing similar platelet inhibition with reduced (60 $\mathrm{mg}$ b.i.d) and standard (90 $\mathrm{mg}$ b.i.d) maintenance doses in stable patients more than 1 year after MI, despite one-third lower ticagrelor plasma concentrations in the lower dose arm [23-25]. Recently, we demonstrated in a randomized, pharmacodynamic trial that a reduced ticagrelor maintenance dose of $60 \mathrm{mg}$ b.i.d. provides a comparable antiplatelet effect to the standard 
$90 \mathrm{mg}$ b.i.d. dose in patients already 1 month post MI, when the disease proceeds to its stable phase. Importantly, no differences in the prevalence of on-ticagrelor high platelet reactivity between patients receiving the reduced and standard maintenance doses was observed in this trial [26, 27].

The number of studies reporting clinical outcomes in coronary artery disease patients receiving reduced maintenance dose of ticagrelor is limited; however, available results indicate that in a stable setting this strategy offers improved safety with preserved efficacy in the prevention of thrombotic events [7]. The PEGASUS-TIMI 54 study showed comparable clinical efficacy of two ticagrelor doses (90 $\mathrm{mg}$ b.i.d. and $60 \mathrm{mg}$ b.i.d.) administered with acetylsalicylic acid (ASA); however, better tolerability of treatment with the lower dose was observed in stable patients 1 year after MI [23].

Another strategy to optimize antiplatelet treatment, by adjusting its potency to time-changing required platelet inhibition, has been validated in the TWILIGHT study [28]. The primary endpoint was the first occurrence of BARC type 2,3 , or 5 bleeding between randomization and 1 year in a time-to-event analysis. The key secondary endpoint was the first occurrence of death from any cause, nonfatal MI, or nonfatal stroke in a time-to-event analysis. The results of this randomized trial showed that in 7119 high-risk patients who had undergone PCI and were treated with ticagrelor $90 \mathrm{mg}$ b.i.d. and ASA for 3 months, subsequent monotherapy with ticagrelor resulted in substantially fewer bleeding events than in the ticagrelor plus ASA arm (4.0\% vs. $7.1 \%$, respectively; HR 0.56 ; $95 \%$ CI $0.45-0.68 ; \mathrm{p}<0.001)$, without any ischemic harm over a period of 1 year; the key secondary endpoint occurred in $3.9 \%$ of patients in both study arms [28]. Even in high-risk patients after complex PCI, the DAPT downgrade to ticagrelor monotherapy was associated with lower incidence of bleeding without increased risk of ischemic events compared with continuation of DAPT [29, 30].

Khan et al. [31] performed a Bayesian network meta-analysis comparing early de-escalation of DAPT (1-3 months) to monotherapy with either P2Y12 inhibitor or ASA versus 12 months deescalation of DAPT after PCI with drug-eluting stent. Among the 7 trials included $(35,821 \mathrm{pa}-$ tients), $52.6 \%$ of patients presented with ACS. No significant differences in terms of ischemic endpoints among different DAPT strategies were found; however, early de-escalation of DAPT to monotherapy with a P2Y12 inhibitor instead of
ASA might be a safer approach compared with 12 months of DAPT in patients treated with PCI [31].

Taking into account these data, we hypothesized that reduction of ticagrelor maintenance dose to $60 \mathrm{mg}$ b.i.d. 1 month after ACS and its continuation until 12 months may improve adherence to antiplatelet treatment due to better tolerability compared with DAPT including standard dose ticagrelor. Moreover, based on the TWILIGHT study results [29], improved safety of treatment and preserved anti-ischemic benefit with additional ASA withdrawal may also be expected. To evaluate these hypotheses, we designed the Evaluating Safety and Efficacy of Two Ticagrelor-based De-escalation Antiplatelet Strategies in Acute Coronary Syndrome - a randomized clinical trial (ELECTRA-SIRIO 2), to assess the influence of ticagrelor dose reduction with or without continuation of ASA versus DAPT with standard-dose ticagrelor in reducing clinically relevant bleeding and maintaining anti-ischemic efficacy in ACS patients.

\section{Current standard of treatment according to the guidelines}

In ACS patients DAPT with a P2Y12 receptor inhibitor on top of ASA is recommended for 12 months to reduce platelet reactivity and adverse thrombotic events (class of recommendation I) $[1,2,4]$. However, in specific clinical scenarios the DAPT duration can be shortened ( $<12$ months), extended (> 12 months), or modified (switching DAPT, DAPT de-escalation), and these decisions depend on individual clinical judgement being driven by the patient's ischemic and bleeding risk, the occurrence of adverse events, comorbidities, co-medications, and availability of drugs [2]. Early discontinuation of $\mathrm{P} 2 \mathrm{Y} 12$ receptor inhibitor therapy 3 months after non-ST-elevation ACS should be considered in those who are at high bleeding risk (High Bleeding Risk according to Academic Bleeding Consortium - ABC-HBR criteria or $\geq 25$ score in PREdicting bleeding Complications In patients undergoing Stent implantation and subsEquent Dual AntiPlatelet Therapy — PRECISE-DAPT score) (class of recommendation IIa) [4]. Termination of treatment with ASA 3-6 months after ACS in patients treated with PCI with stent implantation should be considered, depending on the balance between bleeding and ischemic risk (class of recommendation IIa). Moreover, patients at very high bleeding risk, i.e. those who experienced bleeding episode within a month preceding the index ACS or those scheduled for surgical intervention in the 
early future, may benefit from 1-month DAPT comprising ASA and clopidogrel, with the intention to continue monotherapy with clopidogrel afterwards (class of recommendation IIa) [4].

De-escalation of treatment with P2Y12 receptor inhibitor (e.g. switch from prasugrel or ticagrelor to clopidogrel) may be considered in patients after ACS deemed unsuitable for potent platelet inhibition. De-escalation may be unguided, based solely on clinical judgment or guided by platelet function testing or CYP2C19 genotyping, depending on the patient's risk profile and availability of respective assays (class of recommendation IIb) [4].

Dual antiplatelet treatment with ASA and P2Y12 receptor inhibitor (ticagrelor may be preferred over clopidogrel and prasugrel) or with a low dose of rivaroxaban is recommended in subjects without atrial fibrillation at high/moderate ischemic risk and low bleeding risk beyond 12 months post ACS (class of recommendation IIa/IIb) $[4,5]$.

\section{Adherence to DAPT after ACS}

Despite the numerous advantages of a potent antiplatelet treatment, this therapy is also burdened with non-negligible side effects, greatly related to bleeding. These adverse effects quite often require medical attention or lead to discontinuation of treatment. Of note, the rate of premature discontinuation of antiplatelet treatment in the landmark PLATO study was $23 \%$ of patients receiving ticagrelor and $22 \%$ treated with clopidogrel, which shows the magnitude of the problem [17]. Premature discontinuation of antiplatelet therapy, especially in invasively treated ACS patients, may lead to detrimental cardiovascular and thrombotic events, such as recurrent ACS or stent thrombosis [12, 17-21, 32, 33].

Premature discontinuation of treatment with ticagrelor in the PEGASUS-TIMI 54 was mostly driven by non-serious adverse events that occurred primarily early after randomization, and it was more common in patients receiving standard compared with reduced ticagrelor maintenance dose [23-25]. Thus, better adherence to treatment would be expected with low-dose ticagrelor in real-life practice.

\section{The ELECTRA-SIRIO 2 study}

The ELECTRA-SIRIO 2 study was designed as a phase III, randomized, multicenter, double-blind, placebo-controlled, investigator-initiated clinical study with a 12-month follow-up (Clinical-
Trials.gov Identifier: NCT04718025; EudraCT number: 2020-005130-15). The study is aimed to test two ticagrelor-based de-escalation strategies versus standard treatment.

During the first month after ACS subjects from all three arms will receive a standard DAPT with ticagrelor $90 \mathrm{mg}$ b.i.d and $100 \mathrm{mg}$ ASA once daily. Patients assigned to the control group will continue this standard treatment for a total of 12 months, while in both experimental arms after 1 month the maintenance dose of ticagrelor will be reduced to $60 \mathrm{mg}$ b.i.d. Then, 3 months after ACS, treatment with ASA will be terminated in one of the experimental arms. The primary safety composite endpoint of this study is the first occurrence of type 2,3 , or 5 bleeding according to the BARC criteria, occurring during the first 12 months after ACS. The primary efficacy endpoint is the composite of time to death from any cause, first nonfatal MI, or first nonfatal stroke.

Special care will be applied with regard to adherence to the study treatment (tablet counting at follow-up visits and evaluation with use of the Adherence in Chronic Diseases Scale). The adherence to treatment is of vast importance because early termination of ticagrelor leaves the ACS patients in the monotherapy arm unprotected against ischemic consequences, such as recurrent ACS [34-40].

To increase adherence to treatment all patients will undergo continuous multilevel educational and motivational interventions according to the Multilevel Educational and Motivational Intervention in Patients After Myocardial Infarction (MEDMOTION) project, including assessment with the Readiness for Hospital Discharge after Myocardial Infarction Scale at the end of hospitalization, and with the Functioning in Chronic Illness Scale during follow-ups [41-48].

\section{Summary}

To date, de-escalation of antiplatelet therapy in ACS patients based on lowering the dose of ticagrelor with or without discontinuation of ASA has not been tested in a large randomized clinical trial.

Taking into account existing evidence, one would expect equal clinical efficacy of reduced and standard maintenance doses of ticagrelor already after 1 month post ACS based on their documented antiplatelet effects. The two antiplatelet de-escalation strategies proposed in the ELECTRA-SIRIO 2 study are expected to essentially decrease the incidence of clinically significant bleeding events 
during the first year after ACS, without negative impact on the antithrombotic efficacy. In contrast to the platelet function testing-guided de-escalation strategy applied in the TROPICAL-ACS study [11], the strategy proposed in the ELECTRA-SIRIO 2 study does not require a platelet reactivity assessment, making this step-down approach more feasible for wide application in clinical practice.

Moreover, the quality of life in post-ACS patients on the tested regimen may increase due to lower incidence of dyspnea, an adverse effect typical for ticagrelor. This assumption is based on observations from the PEGASUS-TIMI 54 trial, where dyspnea occurred less frequently in patients who received the lower dose of ticagrelor compared with those treated with the standard dose (16\% vs. 19\%) [23-25]. Thus, a lower number of patients are expected to permanently and prematurely discontinue ticagrelor.

\section{Funding}

This research received financial support from the Medical Research Agency, Poland, Project no. 2019/ABM/01/00009.

Conflict of interest: Jacek Kubica - received lecture honoraria from AstraZeneca; Piotr Adamski - none; Piotr Niezgoda - none; Aldona Kubica - received lecture honoraria from AstraZeneca; Przemysław Podhajski — none; Malwina Barańska - none; Julia M. Umińska - none; Łukasz Pietrzykowski - none; Małgorzata Ostrowska none; Jolanta M. Siller-Matula — received personal fees from Bayer, BMS, Chiesi, and Daiichi, not related to this publication; Jolita Badariene — received an investigator's fee and lecture honoraria from AstraZeneca, investigator's fee from Amgen, and lecture honoraria from Bayer; Stanisław Bartuś — none; Andrzej Budaj - received investigator's fee, advisory board and lecture honoraria from AstraZeneca, Bayer, Bristol Myers Squibb, GlaxoSmithKline, Sanofi Aventis, investigator's fee and lecture honoraria from Novartis, and investigator's fee from Eisai and Amgen; Sławomir Dobrzycki — none; Łukasz Fidor - none; Mariusz Gąsior none; Jacek Gessek — none; Marek Gierlotka none; Robert Gil — none; Jarosław Gorący — none; Paweł Grzelakowski — none; Tomasz Hajdukiewicz - none; Miłosz Jaguszewski - received lecture honoraria from AstraZeneca; Marianna Janion none; Jarosław Kasprzak - received lecture honoraria from AstraZeneca and Bayer; Adam Kern — none; Artur Klecha - none; Andrzej Kleinrok - none; Wacław Kochman - none; Bartosz
Krakowiak — none; Jacek Legutko — received advisory board and lecture honoraria from AstraZeneca and Gedeon Richter; Maciej Lesiak - received speaker and consultancy honoraria from AstraZeneca and Bayer; Marcin Nosal - none; Grzegorz Piotrowski - none; Andrzej Przybylski - none; Tomasz Roleder - none; Grzegorz Skonieczny - none; Grzegorz Sobieszek - none; Agnieszka Tycińska - none; Dariusz Wojciechowski - none; Wojciech Wojakowski - received lecture honoraria from AstraZeneca; Jarosław Wójcik - none; Marzenna Zielińska - none; Aleksander Żurakowski - none; Giuseppe Specchia - none; Diana A. Gorog - received lecture honoraria from AstraZeneca and Boehringer Ingelheim, and a grant from Bayer; Eliano P. Navarese - received grants from Abbott, Amgen, and lecture honoraria from Amgen, AstraZeneca, Bayer, Pfizer, and Sanofi-Regeneron

\section{References}

1. Valgimigli M, Bueno H, Byrne RA, et al. ESC Scientific Document Group, ESC Committee for Practice Guidelines (CPG), ESC National Cardiac Societies. 2017 ESC focused update on dual antiplatelet therapy in coronary artery disease developed in collaboration with EACTS: The Task Force for dual antiplatelet therapy in coronary artery disease of the European Society of Cardiology (ESC) and of the European Association for CardioThoracic Surgery (EACTS). Eur Heart J. 2018; 39(3): 213-260, doi: 10.1093/eurheartj/ehx419, indexed in Pubmed: 28886622.

2. Neumann FJ, Sousa-Uva M, Ahlsson A, et al. ESC Scientific Document Group. 2018 ESC/EACTS Guidelines on myocardial revascularization. Eur Heart J. 2019; 40(2): 87-165, doi: 10.1093/ eurheartj/ehy394, indexed in Pubmed: 30165437.

3. Adamski P, Adamska U, Ostrowska M, et al. New directions for pharmacotherapy in the treatment of acute coronary syndrome. Expert Opin Pharmacother. 2016; 17(17): 2291-2306, doi: 10.1080/14656566.2016.1241234, indexed in Pubmed: 27677394.

4. Collet JP, Thiele H, Barbato E, et al. ESC Scientific Document Group. 2020 ESC Guidelines for the management of acute coronary syndromes in patients presenting without persistent ST-segment elevation. Eur Heart J. 2021; 42(14): 1289-1367, doi: 10.1093/eurheartj/ehaa575, indexed in Pubmed: 32860058.

5. Kubica J, Adamski P, Niezgoda P, et al. Prolonged antithrombotic therapy in patients after acute coronary syndrome: A critical appraisal of current European Society of Cardiology guidelines. Cardiol J. 2020; 27(6): 661-676, doi: 10.5603/CJ.a2020.0132, indexed in Pubmed: 33073857.

6. Adamski P, Adamska U, Ostrowska M, et al. Evaluating current and emerging antithrombotic therapy currently available for the treatment of acute coronary syndrome in geriatric populations. Expert Opin Pharmacother. 2018; 19(13): 1415-1425, doi: 10.1080/14656566.2018.1510487, indexed in Pubmed: 30132731.

7. Claassens DMf, Sibbing D. De-escalation of antiplatelet treatment in patients with myocardial infarction who underwent percutaneous coronary intervention: a review of the current literature. J Clin Med. 2020; 9(9), doi: 10.3390/jcm9092983, indexed in Pubmed: 32942754. 
8. Kubica J, Adamski P, Paciorek P, et al. Treatment of patients with acute coronary syndrome: Recommendations for medical emergency teams: Focus on antiplatelet therapies. Updated experts' standpoint. Cardiol J. 2018; 25(3): 291-300, doi: 10.5603/ CJ.a2018.0042, indexed in Pubmed: 29671864.

9. Cuisset T, Deharo P, Quilici J, et al. Benefit of switching dual antiplatelet therapy after acute coronary syndrome: the TOPIC (timing of platelet inhibition after acute coronary syndrome) randomized study. Eur Heart J. 2017; 38(41): 3070-3078, doi: 10.1093/eurheartj/ehx175, indexed in Pubmed: 28510646.

10. De Luca L, D’Ascenzo F, Musumeci G, et al. Incidence and outcome of switching of oral platelet P2Y12 receptor inhibitors in patients with acute coronary syndromes undergoing percutaneous coronary intervention: the SCOPE registry. EuroIntervention. 2017; 13(4): 459-466, doi: 10.4244/EIJ-D-17-00092, indexed in Pubmed: 28374678.

11. Sibbing D, Aradi D, Jacobshagen C, et al. Guided de-escalation of antiplatelet treatment in patients with acute coronary syndrome undergoing percutaneous coronary intervention (TROPICAL-ACS): a randomised, open-label, multicentre trial. Lancet. 2017; 390(10104): 1747-1757, doi: 10.1016/s0140-6736(17)32155-4.

12. Navarese EP, Khan SU, Kołodziejczak M, et al. Comparative efficacy and safety of oral P2Y inhibitors in acute coronary syndrome: network meta-analysis of 52816 patients from 12 randomized trials. Circulation. 2020; 142(2): 150-160, doi: 10.1161/CIRCULATIONAHA.120.046786, indexed in Pubmed: 32468837 .

13. Kheiri B, Osman M, Abdalla A, et al. De-Escalation of antiplatelet therapy in patients with acute coronary syndrome undergoing percutaneous coronary intervention: a meta-analysis of randomized clinical trials. J Cardiovasc Pharmacol Ther. 2019; 24(2): 153-159, doi: 10.1177/1074248418809098, indexed in Pubmed: 30419754 .

14. Kubica J, Adamski P, Ostrowska M, et al. Morphine delays and attenuates ticagrelor exposure and action in patients with myocardial infarction: the randomized, double-blind, placebo-controlled IMPRESSION trial. Eur Heart J. 2016; 37(3): 245-252, doi: 10.1093/eurheartj/ehv547, indexed in Pubmed: 26491112.

15. Hobl EL, Reiter B, Schoergenhofer C, et al. Morphine decreases ticagrelor concentrations but not its antiplatelet effects: a randomized trial in healthy volunteers. Eur J Clin Invest. 2016; 46(1): 7-14, doi: 10.1111/eci.12550, indexed in Pubmed: 26449338.

16. Kubica J, Kubica A, Jilma B, et al. Impact of morphine on antiplatelet effects of oral P2Y12 receptor inhibitors. Int J Cardiol. 2016; 215: 201-208, doi: 10.1016/j.ijcard.2016.04.077, indexed in Pubmed: 27128531.

17. Adamski P, Ostrowska M, Navarese EP, et al. Pharmacodynamic and clinical efficacy of reduced ticagrelor maintenance doses in patients with coronary artery disease. Curr Med Res Opin. 2021; 37(2): 195-206, doi: 10.1080/03007995.2020.1854207, indexed in Pubmed: 33211543.

18. Adamski P, Buszko K, Sikora J, et al. Determinants of high platelet reactivity in patients with acute coronary syndromes treated with ticagrelor. Sci Rep. 2019; 9(1): 3924, doi: 10.1038/s41598019-40628-0, indexed in Pubmed: 30850677.

19. Ostrowska M, Kubica J, Adamski P, et al. Stratified approaches to antiplatelet therapies based on platelet reactivity testing. Front Cardiovasc Med. 2019; 6: 176, doi: 10.3389/fcvm.2019.00176, indexed in Pubmed: 31850373.

20. Adamski P, Sikora J, Laskowska E, et al. Comparison of bioavailability and antiplatelet action of ticagrelor in patients with
ST-elevation myocardial infarction and non-ST-elevation myocardial infarction: A prospective, observational, single-centre study. PLoS One. 2017; 12(10): e0186013, doi: 10.1371/journal. pone.0186013, indexed in Pubmed: 29023473.

21. Wallentin L, Becker RC, Budaj A, et al. Ticagrelor versus clopidogrel in patients with acute coronary syndromes. N Engl J Med. 2009; 361(11): 1045-1057, doi: 10.1056/NEJMoa0904327, indexed in Pubmed: 19717846.

22. Wiviott S, Braunwald E, McCabe C, et al. TRITON-TIMI 38 Investigators. Prasugrel versus copidogrel in patients with acute coronary syndromes. N Engl J Med. 2007; 357(20): 2001-2015, doi: 10.1056/nejmoa0706482, indexed in Pubmed: 17982182.

23. Bonaca MP, Bhatt DL, Cohen M, et al. PEGASUS-TIMI 54 Steering Committee and Investigators. Long-term use of ticagrelor in patients with prior myocardial infarction. N Engl J Med. 2015; 372(19): 1791-1800, doi: 10.1056/NEJMoa1500857, indexed in Pubmed: 25773268.

24. Storey RF, Angiolillo DJ, Bonaca MP, et al. Platelet inhibition with ticagrelor $60 \mathrm{mg}$ versus $90 \mathrm{mg}$ twice daily in the PEGASUSTIMI 54 trial. J Am Coll Cardiol. 2016; 67(10): 1145-1154, doi: 10.1016/j.jacc.2015.12.062, indexed in Pubmed: 26965534.

25. Bonaca MP, Bhatt DL, Oude Ophuis T, et al. Long-term tolerability of ticagrelor for the secondary prevention of major adverse cardiovascular events: a secondary analysis of the PEGASUS-TIMI 54 trial. JAMA Cardiol. 2016; 1(4): 425-432, doi: 10.1001/ jamacardio.2016.1017, indexed in Pubmed: 27438319.

26. Kubica J, Adamski P, Buszko K, et al. Rationale and Design of the Effectiveness of LowEr maintenanCe dose of TicagRelor early After myocardial infarction (ELECTRA) pilot study. Eur Heart J Cardiovasc Pharmacother. 2018; 4(3): 152-157, doi: 10.1093/ ehjcvp/pvx032, indexed in Pubmed: 29040445.

27. Kubica J, Adamski P, Buszko K, et al. Platelet inhibition with standard vs. lower maintenance dose of ticagrelor early after myocardial infarction (ELECTRA): a randomized, open-label, active-controlled pharmacodynamic and pharmacokinetic study. Eur Heart J Cardiovasc Pharmacother. 2019; 5(3): 139-148, doi: 10.1093/ehjcvp/pvz004, indexed in Pubmed: 30689800.

28. Mehran R, Baber U, Sharma S, et al. Ticagrelor with or without Aspirin in High-Risk Patients after PCI. N Engl J Med. 2019; 381(21): 2032-2042, doi: 10.1056/nejmoa1908419, indexed in Pubmed: 31556978.

29. Dangas G, Baber U, Sharma S, et al. Ticagrelor with or without aspirin after complex PCI: the TWILIGHT-COMPLEX analysis. J Am Coll Cardiol. 2020; 75(19): 2414-2424, doi: 10.1016/j. jacc.2020.03.011, indexed in Pubmed: 32240761.

30. Gelbenegger G, Schoergenhofer C, Jilma B, et al. Efficacy and safety of ticagrelor monotherapy in patients undergoing percutaneous coronary intervention: a meta-analysis. Clin Pharmacol Ther. 2021 [Epub ahead of print], doi: 10.1002/cpt.2226, indexed in Pubmed: 33668076.

31. Khan SU, Khan MZ, Khan MS, et al. De-escalation of antiplatelets after percutaneous coronary intervention: a bayesian network meta-analysis of various de-escalation strategies. Eur Heart J Cardiovasc Pharmacother. 2020 [Epub ahead of print], doi: 10.1093/ehjcvp/pvaa025, indexed in Pubmed: 32271872.

32. Pietrzykowski $€$, Kasprzak M, Michalski P, et al. Therapy discontinuation after myocardial infarction. J Clin Med. 2020; 9(12): 4109, doi: 10.3390/jcm9124109, indexed in Pubmed: 33352811.

33. Kołodziejczak M, Navarese E, Kubica J. Rationale and design of PREvalence of DyspneA in patients treated with TicagreIOR (PREDATOR) program. Med Res J. 2018, doi: 10.5603/mrj. a2018.0037. 
34. Kubica A, Obońska K, Fabiszak T, et al. Adherence to antiplatelet treatment with $\mathrm{P} 2 \mathrm{Y} 12$ receptor inhibitors. Is there anything we can do to improve it? A systematic review of randomized trials. Curr Med Res Opin. 2016; 32(8): 1441-1451, doi: 10.1080/03007995.2016.1182901, indexed in Pubmed: 27112628.

35. Kubica A, Kasprzak M, Obońska K, et al. Discrepancies in assessment of adherence to antiplatelet treatment after myocardial infarction. Pharmacology. 2015; 95(1-2): 50-58, doi: 10.1159/000371392, indexed in Pubmed: 25592409.

36. Kubica A. Self-reported questionnaires for a comprehensive assessment of patients after acute coronary syndrome. Med Res J. 2019; 4(2): 106-109, doi: 10.5603/mrj.a2019.0021.

37. Kosobucka A, Michalski P, Pietrzykowski Ł, et al. The impact of readiness to discharge from hospital on adherence to treatment in patients after myocardial infarction. Cardiol J. 2020 [Epub ahead of print], doi: 10.5603/CJ.a2020.0005, indexed in Pubmed: 32037501.

38. Kubica A, Kosobucka A, Fabiszak T, et al. Assessment of adherence to medication in patients after myocardial infarction treated with percutaneous coronary intervention. Is there a place for newself-reported questionnaires? Curr Med Res Opin. 2019; 35(2): 341-349, doi: 10.1080/03007995.2018.1510385, indexed in Pubmed: 30091642.

39. Kosobucka A, Michalski P, Pietrzykowski $Ł$, et al. Adherence to treatment assessed with the Adherence in Chronic Diseases Scale in patients after myocardial infarction. Patient Prefer Adherence. 2018; 12: 333-340, doi: 10.2147/PPA.S150435, indexed in Pubmed: 29551891.

40. Pietrzykowski $Ł$, Michalski P, Kosobucka A, et al. Medication adherence and its determinants in patients after myocardial infarction. Sci Rep. 2020; 10(1): 12028, doi: 10.1038/s41598-02068915-1, indexed in Pubmed: 32694522.

41. Kosobucka A, Pietrzykowski $€$, Michalski P, et al. Impact of readiness for discharge from the hospital on the implementation of the therapeutic plan. Med Res J. 2020; 5(4): 256-264, doi: 10.5603/mrj.a2020.0047.

42. Michalski P, Kasprzak M, Siedlaczek M, et al. The impact of knowledge and effectiveness of educational intervention on readiness for hospital discharge and adherence to therapeutic recommendations in patients with acute coronary syndrome. Med Res J. 2020, doi: 10.5603/mrj.a2020.0023.

43. Kubica A, Kosobucka A, Michalski P, et al. Self-reported questionnaires for assessment adherence to treatment in patients with cardiovascular diseases. Med Res J. 2018; 2(4): 115-122, doi: 10.5603/mrj.2017.0015.

44. Kubica A, Gruchała M, Jaguszewski M, et al. Adherence to treatment - a pivotal issue in long-term treatment of patients with cardiovascular diseases. An expert standpoint. Med Res J. 2018; 2(4): 123-127, doi: 10.5603/mrj.2017.0016.

45. Buszko K, Pietrzykowski Ł, Michalski P, et al. Validation of the Functioning in Chronic Illness Scale (FCIS). Med Res J. 2018; 3(2): 63-69, doi: 10.5603/mrj.2018.0011.

46. Pietrzykowski $€$, Michalski P, Kosobucka A, et al. Knowledge about health and disease in obese patients after myocardial infarction. An observational study. Med Res J. 2018; 2(4): 135-140, doi: 10.5603/mrj.2017.0018.

47. Buszko K, Kosobucka A, Michalski P, et al. The readiness for hospital discharge of patients after acute myocardial infarction: a new self-reported questionnaire. Med Res J. 2017; 2(1): 20-28, doi: 10.5603/mrj.2017.0004.

48. Kubica A, Adamski P, Bączkowska A, et al. The rationale for Multilevel Educational and Motivational Intervention in Patients after Myocardial Infarction (MEDMOTION) project is to support multicentre randomized clinical trial Evaluating Safety and Efficacy of Two Ticagrelor-based De-escalation Antiplatelet Strategies in Acute Coronary Syndrome (ELECTRA-SIRIO 2). Med Res J. 2020; 5(4): 244-249, doi: 10.5603/mrj.a2020.0043. 